\title{
HARDWARE IMPLEMENTATION OF ARTIFICIAL NEURAL NETWORKS USING BACK PROPAGATION ALGORITHM ON FPGA
}

\author{
Chaitra.P $\mathbf{P}^{1}$ \\ ${ }^{I}$ Electronics and Communication Engineering, Department ECE, Sapthagiri College of Engineering
}

\begin{abstract}
In order to handle problems such as massive parallelism, Fault tolerance, self learning, adaptivity, computational complexity researchers have developed intelligent system such as artificial neural networks. ANN(Artificial neural network) addresses the issues related to pattern recognition, prediction, associative memory and control. It mimics the human biological neural network and has a human like learning ability and is inspired by its structure, processing method and its learning ability like a human brain. Different algorithms are proposed by the designers to train the neural networks, among those Back propagation algorithm in its gradient descent form is widely used algorithm which provides better performance. Verilog coding is done for ANN and Back propagation training algorithm. The functionality of Verilog is verified by simulation using ModelsimSE 6.3F Simulator. The Verilog code is synthesized using Xilinx ISE 14.7 tool. Finally ANN and Back propagation algorithm was successfully implemented.
\end{abstract}

Keywords: ANN, Back propagation algorithm, Fuzzy logic, Synapses

\section{INTRODUCTION}

ANN also known as neural networks are the systems which has human like learning ability. It replicates the human biological neuron system. The gained information is stored in internal elements called synapses. It can be used for classification, pattern recognition, weather forecasting, medical science etc. ANN finds major application in the field of image processing. Very complex relation between the input set and the output set can be handled using ANN. They also have the advantages of self learning, adapt easy to the situations, flexibility which make them suitable for noise classification. Many algorithms are proposed to train the ANN among those $\mathrm{BPN}(\mathrm{Back}$ propagation algorithm) is widely used. In BPN the errors are propagated in backward direction and the weights are updated. Weight Updation may take several iterations until the difference in the actual and obtained value becomes zero. Neuro-fuzzy model are neural network based fuzzy system where ANN is used for processing of data and fuzzy sets are used to handle the randomness. They are also used in image processing for various applications such as classification, enhancement, edge detection, segmentation. The fuzzy sets are controlled using neural networks with a pair of inputs and outputs. First the system behaves as ANN which is trained with suitable algorithm and at execution time it behaves like fuzzy. Generally the neural chip is implemented using ANN which is trained using software but this makes the chip fixed without further modifications. In order to overcome this limitation learning algorithm in ANN can be implemented using hardware. The selection of training algorithm depends on hardware implementation, so training sets which occupies less FPGA area is most suitable. So gradient descent BPN in its steepest form is considered. The FPGA implementation of the proposed method gives better performance compared to the existing methodologies.

\section{DETAILS EXPERIMENTAL}

\subsection{Artificial Neural Network}

ANN is the model which computes the complex relationship between inputs and the outputs. It is a non-linear modeling tool. The basic computing elements are neurons. They are responsible for processing of information through interconnection. They are non-linear data modeling tools which are used for patterning the data, classification etc. There are basically two types of learning algorithm: Supervised learning and unsupervised learning. In supervised learning the inputs and outputs are known prior to the computation whereas in unsupervised learning is similar to learning without a teacher. From the below figure it can be seen that the neural network consists of input layer, hidden layer and output layer. Initially all the calculations from the input sets are carried towards the output layer through the hidden layer. Each neuron is a combination of a summer and a activation function. Initially the inputs from the input layer are multiplied with the weights and given to the hidden layer where the multiplied values are summed up and given to activation function. In the proposed model sigmoidal activation function is used

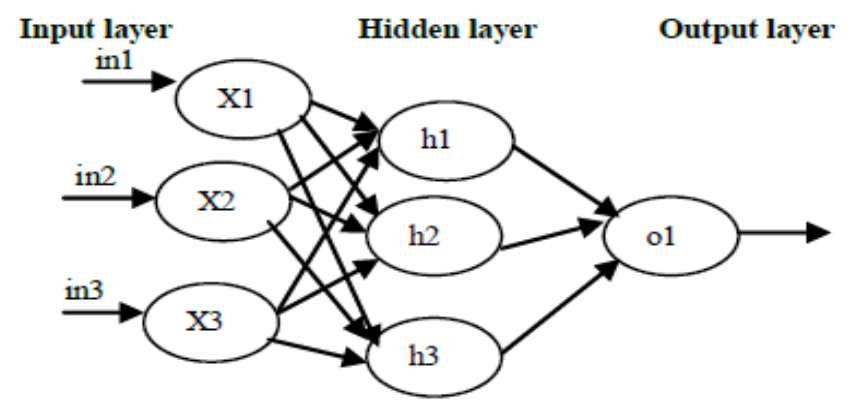

Fig 1: Neural network structure. 


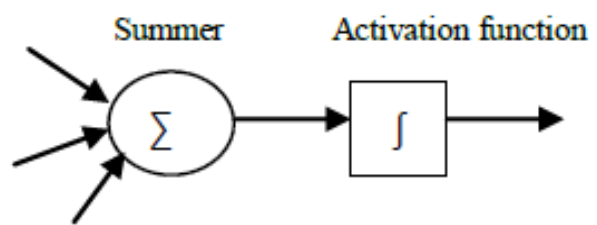

Fig 2: A neuron model

\subsection{Back Propagation Training Algorithm}

It is a supervised learning algorithm where the input and output are known prior to the computation. The signals from the input layer propagate to the output layer through the hidden neurons in a feed forward manner, the output is compared with the expected value and the difference in the error is back propagated making the weights to be updated. There are basically two ways of propagation: A forward path and a backward path. In forward path the stnaptic weights are fixed and signals moves in forward direction whereas in backward path the weights are adjusted according to the calculated error. The difference in the actual and obtained values of output are measured the error is propagated backwards hence the name "Back propagation". The BP algorithm is implemented in its gradient descent form in which the weights are selected so as to reduce the error.

\section{Change in Weight:}

$$
\begin{aligned}
& \Delta W_{k j}=\varepsilon \delta_{k} a_{i} \\
& \Delta W_{k j}=\text { =change in weights, } \\
& a_{i}=\text { input value } \\
& \delta_{k j}=\text { Actual value-obtained value, }
\end{aligned}
$$

\section{Updated Weight:}

$$
W_{k j}(n+1)=W_{k j}(n)+\Delta W_{k j}
$$

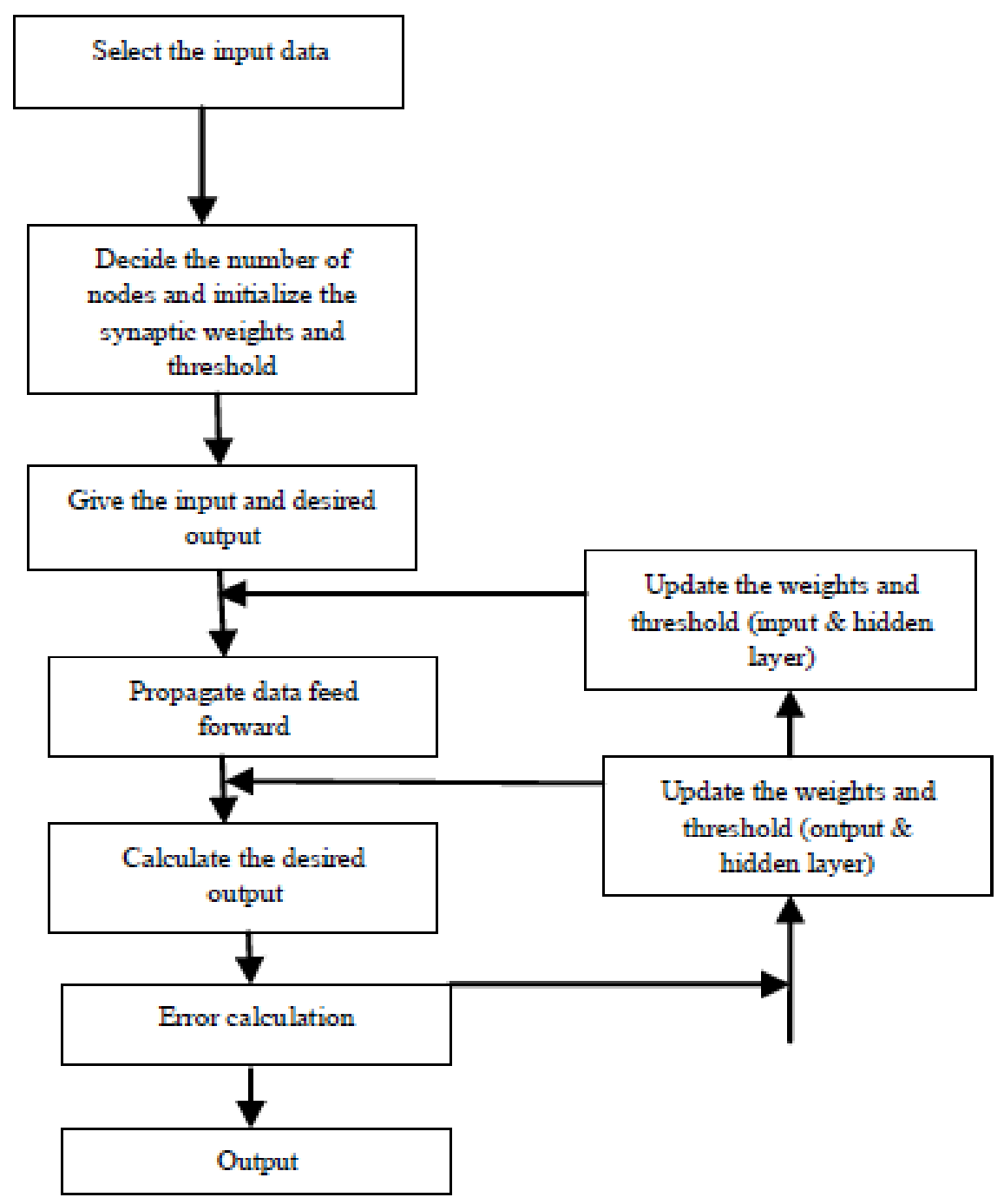

Fig 3: Architecture of BPN 


\section{RESULTS AND DISCUSSION}

\subsection{Simulation Results of 4:5:1 ANN using BP Training Algorithm}

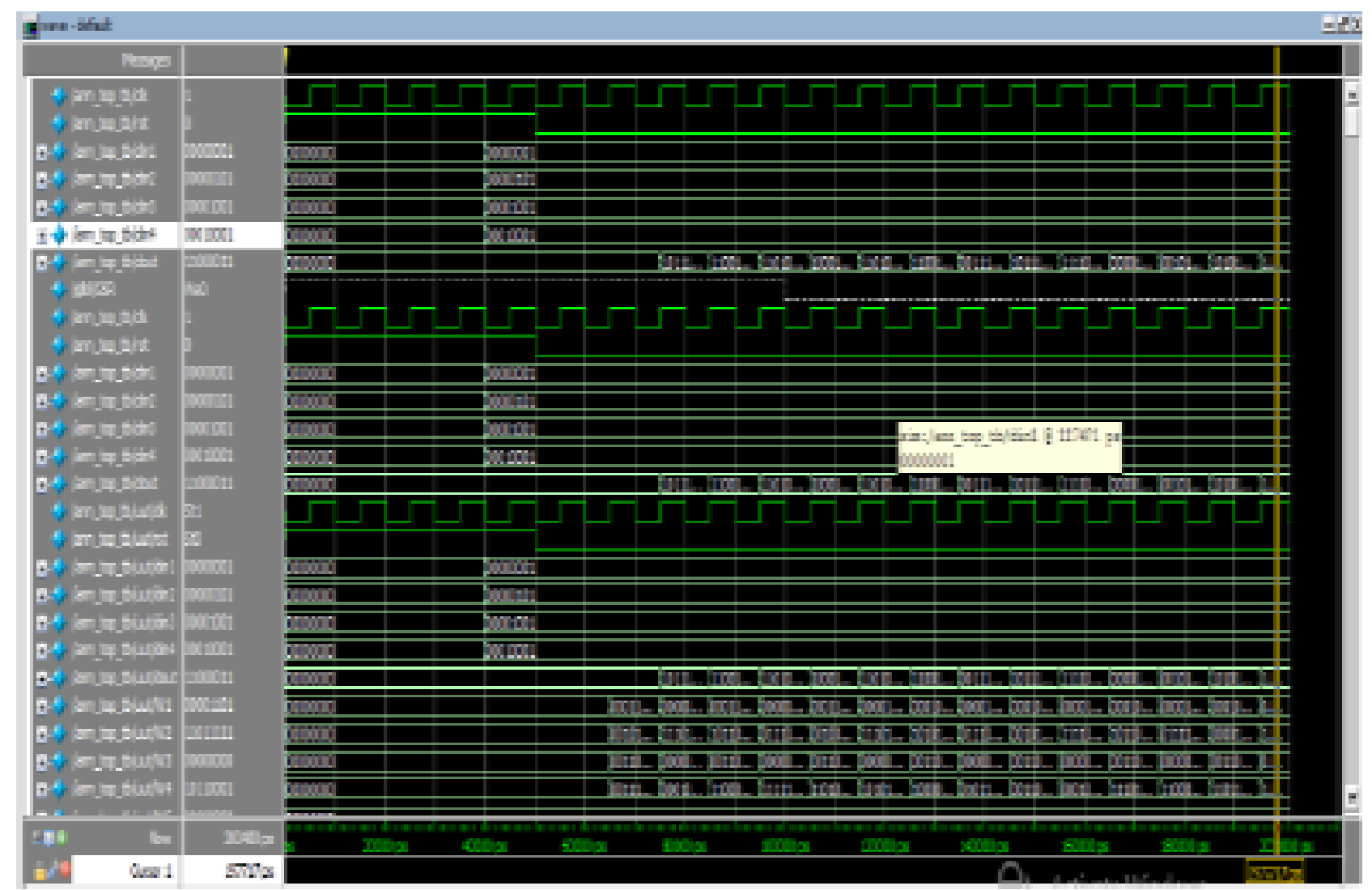

The above figure shows the simulation output of neural network with 4 input set $\operatorname{din} 1, \operatorname{din} 2$, din3, din 4 and clock, reset. When reset is high the output is zero and when reset is low the output dout produces some output. The obtained result is compared with actual value and if any error occurs the weight are updated and is propagated forward direction.

\subsection{Table 1: Device Utilization Summary of ANN}

\section{for the Device xc3s400-5pq208, Spartan 3E Family}

\begin{tabular}{|c|c|c|c|}
\hline $\begin{array}{c}\text { Logic } \\
\text { utilization }\end{array}$ & Used & Available & Utilization \\
\hline $\begin{array}{c}\text { Number of } \\
\text { slices }\end{array}$ & 788 & 3594 & $21 \%$ \\
\hline $\begin{array}{c}\text { Number of } \\
\text { slice Flip } \\
\text { flop }\end{array}$ & 228 & 7168 & $3 \%$ \\
\hline $\begin{array}{c}\text { Number of } \\
4 \text { input } \\
\text { LUTs }\end{array}$ & 1470 & 7168 & $20 \%$ \\
\hline $\begin{array}{c}\text { Number of } \\
\text { bonded } \\
\text { IOBs }\end{array}$ & 42 & 141 & $29 \%$ \\
\hline $\begin{array}{c}\text { Number of } \\
\text { MULT } \\
18 x 18 \text { s }\end{array}$ & 16 & 16 & $100 \%$ \\
\hline $\begin{array}{c}\text { Number of } \\
\text { GCLKs }\end{array}$ & 2 & 8 & $25 \%$ \\
\hline
\end{tabular}

\section{CONCLUSION AND FUTURE SCOPE}

Artificial neural network using back propagation algorithm overcomes various challenges. It is possible to handle larger data set and complex network using ANN. They also provide data security by the use of more hidden layers. Fuzzy are the excellent means of enhancing the image because they can handle the uncertainties using fuzzy rules. Neuro-fuzzy model can be used in various fields of data processing, image processing and also in control system applications

\section{ACKNOWLEDGMENT}

ANN is better field for various application. FPGA implementation provides ANN to handle parallel architectures and makes neural network reconfigurable. BPN are widely used algorithm for training which provides better means for classification purpose. They are also upcoming trends in image processing for image enhancement, image noise classification etc.

\section{REFERENCES}

[1]. Nieuwenhuis, Claudia, and Michelle Yan, Knowledge based image enhancement using neural networks, 18th International Conference on Pattern Recognition, vol. 3, IEEE 2006, pp. 814-817. 
[2]. B. Verma, M. Blumenstein and S. Kulkarni, A Neural Network based Technique for Data Compression, Griffith University, Gold Coast Campus, Australia.

[3]. Venkata Rama Prasad Vaddella, Kurupati Rama, Artificial Neural Networks For Compression Of Digital Images: A Review, International Journal of Reviews in Computing, 2009-2010.

[4]. S. L. Pinjare, Arun Kumar M., Implementation of Neural Network Back Propagation Training Algorithm on FPGA, International Journal of Computer Applications (0975 - 8887) Volume 52- No.6, August 2012.

[5]. L. Zadeh, Fuzzy Sets, Information Control, 1965 pp. 338-353.

[6]. Tarun Mahashwari, Amit Asthana, International Journal of Research Review In Engineering Science \& Technology (ISSN 2278-6643) Volume-2, Issue-2, June-2013.

[7]. Thiang, Handry Khoswanto, Rendy Pangaldus, Artificial Neural Network with Steepest Descent Backpropagation Training Algorithm for Modeling Inverse Kinematics of Manipulator, World Academy of Sciences, Engineering and Technology, 2009.

[8]. P.B Khanale and S.D Chitnis,2011.Handwritten Devangiri Character Recognition sing Artificial neural network. Journal of Artificial intelligence. 